\title{
A MODEL FOR THE ESTIMATION OF ROAD ROUGHNESS CONDITION FROM SENSOR DATA COLLECTED BY ANDROID SMARTPHONES
}

\author{
Viengnam DOUANGPHACHANH ${ }^{1}$ and Hiroyuki ONEYAMA ${ }^{2}$ \\ ${ }^{1}$ Member of JSCE, Ph.D. Student, Dept. of Civil and Environmental Eng., Tokyo Metropolitan University \\ (1-1 Minami Osawa, Hachioji-shi, Tokyo 192-0397, Japan) \\ E-mail: douangphachanh-viengnam@ed.tmu.ac.jp \\ ${ }^{2}$ Member of JSCE, Professor, Dept. of Civil and Environmental Eng., Tokyo Metropolitan University \\ (1-1 Minami Osawa, Hachioji-shi, Tokyo 192-0397, Japan) \\ E-mail: oneyama@tmu.ac.jp
}

\begin{abstract}
Road roughness condition information is very important for the maintenance planning and management of road infrastructure. Collecting such information is time and resource consuming because it usually requires either, if not all, skillful manpower, substantial budget, and sophisticated equipment. Therefore, collecting and updating road roughness condition is viewed as a big challenge for many road authorities. The final goal of our research is to explore for a low cost and easy to use approach for the aforementioned purpose by using Android smartphones to estimate road roughness and traffic conditions. In this study we develop a model based on a relationship function of smartphone sensor data and road roughness condition. The relationship function is drawn from our experiment in Vientiane, Laos, in which we have found that the acceleration data from smartphones has a linear relationship with road roughness condition and the significant of the relationship also partly depends on speed. The most important and unique feature of the model is the ability to estimate road roughness condition using data obtained from anonymous drivers. Preliminary numerical examples and simulations using real sensor data have been carried out whereas the findings show that performance of the model and results are very promising.
\end{abstract}

Key Words : road roughness, roughness condition estimation, smartphone sensor data, estimation model

\section{INTRODUCTION}

Road roughness condition is the irregularity in the road surface that usually occurs due to the deterioration of the road surface over time, the type of the surface material, and quality of the pavement work. Bad roughness condition may cause uncomfortable ride, extra fuel consumption, unexpected vehicle maintenance costs and safety. Road infrastructure maintenance planning and management is very important to keep the infrastructure in good condition, so that it does not affect road users especially in terms of vehicle maintenance, fuel consumption and ride quality. In developing nations, particularly, maintaining a good quality road infrastructure is a big challenge for almost all road authorities. International Roughness Index (IRI) is an indicator that is widely adopted as a measurement for road surface roughness condition ${ }^{1)}$. IRI is the condition index obtained from the measurement of longitudinal road profiles with the measuring unit of slope $(\mathrm{mm} / \mathrm{m}, \mathrm{m} / \mathrm{km}$ for instance). To obtaining IRI, which is a crucial piece of information for the maintenance and management planning, there are 2 main approaches. The first approach is subjective inspection and the other approach is the use of sophisticated profilers. The former approach is labor intensive and very time consuming because it relies mainly on manual inspection by the inspectors. Accuracy is also subjective to the judgment of the inspectors, which could be inconsistent if not well or properly trained. The later approach relies on the use of one or many of various road profilers available in the market. This approach takes less time, but requires considerably investment especially to obtain, operate and maintain such profilers. Skillful operators are also recommended. Additionally, in order to properly use such profilers, majority of them would require cumbersome calibration before deployment.

The final goal of our research is to explore for a low cost and easier way for continuous road condition monitoring by obtaining road surface (roughness) and traffic conditions data using Android smartphones. We believe smartphones with a proper app would help road authorities in overcoming some difficulties relat- 


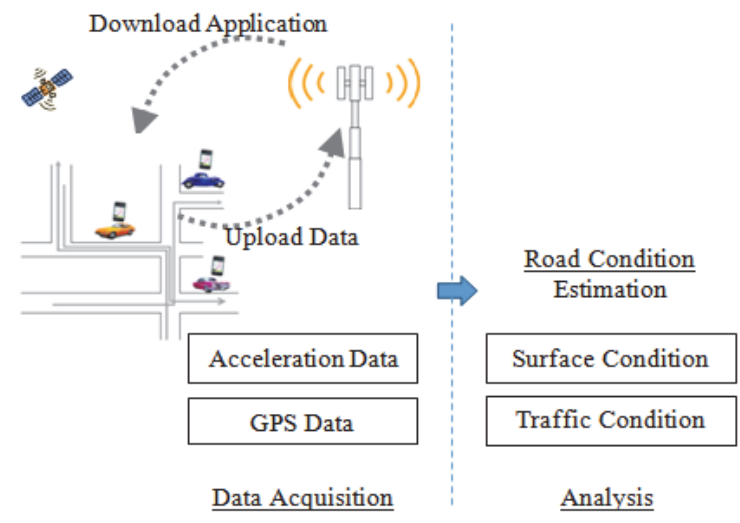

Fig.1 Conceptual image of continuous road condition monitoring system.

ing to roughness and traffic condition data collection and updating. Basic concept of our approach is shown in Fig. 1 above.

This paper focuses on the development of a simple model to estimate road roughness condition from acceleration and speed data. In our upcoming studies, the model is expected to be used for the development of a smartphone app to estimate road roughness condition. The approach will be positioned as an estimation instrument that is carried out by anonymous drivers on the actual traffic. Despite the fact that a single estimation from an individual or anonymous driver may not be accurate, in comparison to the use of profilers or VIMS, for instance; with increased or accumulated estimations being made available by many drivers would enable the approach to improve the accuracy of the estimation to an acceptable level needed for the purpose of road infrastructure maintenance planning and management.

\section{RELATED WORK}

Recently, there are some groups of researchers that are interested in detecting road bumps and anomalies using mobile sensors. However, majority of these researchers put a lot of their focuses on identifying and locating road bumps and anomalies instead of estimating IRI based road surface condition. Gonzalez et $a{ }^{2}{ }^{2}$ use a standalone accelerometer to fit in a simulation car and use it to assess road roughness condition. Their simulations conclude that roughness of the road can be estimated from acceleration data obtained from the sensor. Similarly, in another study, a system has been developed to utilize standalone accelerometers to successfully detect road anomalies ${ }^{3)}$. In India, a group of researchers use many sensing component from mobile phone such as accelerometer, microphone, Global System for Mobile communication (GSM) radio, and Global Positioning System (GPS) to monitor road and traffic condition ${ }^{4)}$. By an- alyzing data from the sensors, potholes, bumps, braking and honking can be detected. The information is then used to assess road and traffic conditions. Mednis et $a l^{5)}$ and Strazdins et $a l^{6}{ }^{6}$ use Android smartphone devices with accelerometer to detect location of potholes. Their approach includes many simple algorithms to detect events in the acceleration vibration data. Tai et al. ${ }^{7)}$ and Perttunen et al. ${ }^{8)}$ analyze data obtained by smartphone accelerometers in frequency domain to extract features that are corresponding to road bumps. In Japan, a group of researchers has developed an Android smartphone application called "BumpRecorder" to detect the location and severity of road bumps on road network that have been affected by the March 11 earthquake in Tohoku region, $\operatorname{Japan}^{9)}$. This app, however, is not able to evaluate road roughness, particularly estimating IRI based condition. "Roadroid"10) is another app that has been developed with a main purpose of being an instrument to estimate road roughness condition. This app can assess IRI based roughness condition, however it still have some limitations in the sense that it requires smartphones to be fixed at one location and orientation. The app also needs to be calibrated before use.

Vehicle Intelligent Monitoring System (VIMS) is a system that has been developed with a purpose of being a low cost solution or choice for road authorities to monitor their road networks ${ }^{11,12), 13)}$. An original VIMS consists of accelerometer and many other component that are connected to each other via cables. A further development has introduced a smartphone gyroscope to help redefine VIMS ${ }^{14)}$. Despite many merits, there are still some limitations that are hindering the use of it. Main limitations include the need for 2 separate lengthy calibrations and its component setting, which is still practically inconvenient for the users ${ }^{14}$.

As already mentioned, our final goal is to develop a system that minimizes the above mentioned limitations, the smartphone app to be developed in our forthcoming studies is expected to be much simpler and convenient for users. Calibration and the restriction on location and orientation of the smartphones should be no longer needed. Importantly, the app shall have an estimation model that takes advantages of data being collected and analyzed by anonymous drivers.

\section{EXPERIMENT}

To realize our model formulation, first, the relationship between road roughness condition and smartphone sensor data must be studied. An experiment has been conducted in Vientiane, Laos in November, 2012; where we use 4 smartphones to place at different location inside 4 experiment vehicles (one vehicle 


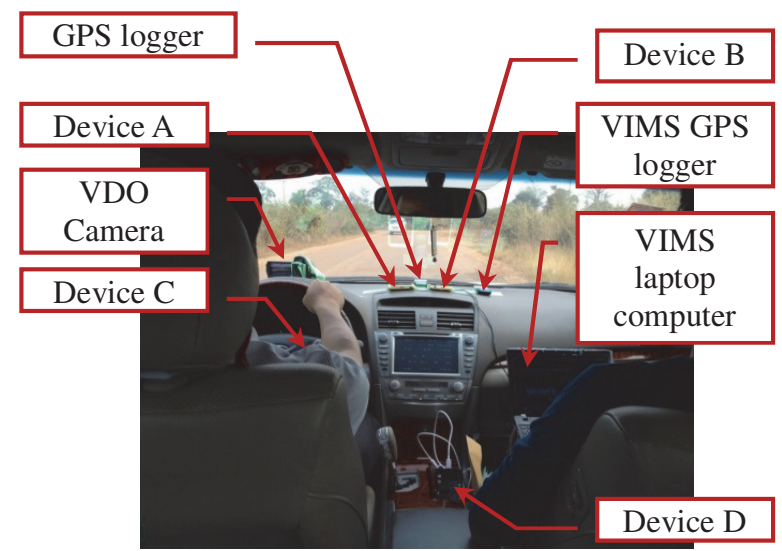

Fig.2 Equipment setting.

at a time). All 4 smartphones are pre-installed with an application called AndroSensor ${ }^{15)}$. For this experiment, only acceleration data $(x, y, z)$ from accelerometer and location data (including speed) from GPS are needed. Data recording is done at an interval of 0.01 second or at a frequency rate of $100 \mathrm{~Hz}$.

VIMS has been used to collect the referenced pavement condition data for this study. The system measures IRI of the road pavement for every 10 meter road section (VIMS section).

The 4 devices (smartphones) are placed at different locations inside the vehicles. Device A and Device B are glued closed to each other on the dashboard of the experiment vehicles with strong and thin adhesive tapes (Fixed devices). Device $\mathrm{C}$ is placed inside the driver's shirt pocket and Device D is placed at a box near the vehicles' gear handle. Device C and Device $\mathrm{D}$ are assumed to be free (not fixed) as they can move more freely. Other equipment such as GPS and video camera are also placed on the dash board. VIMS components are also installed in accordance to the VIMS manual ${ }^{16)}$.

The data, collected from smartphones, is checked, matched with referenced data, and sectioned into 100 $\mathrm{m}$ sections. Analysis is carried out in frequency domain to calculate magnitudes of acceleration data for each $100 \mathrm{~m}$ section using Fast Fourier Transform (FFT). The average IRI is also calculated for each $100 \mathrm{~m}$ section, which is the average IRI of the corresponding 10 VIMS sections. Details of the analysis are discussed in our previous papers ${ }^{17), 18), 19)}$. Selected results are presented below.

From the analysis, it has been found that acceleration data from smartphones has linear relationship with road roughness condition. Selected results in Fig. 3 show the relationships $\left(R^{2}\right)$ for a device in each vehicle. The $R^{2}$ values are slightly different depending on settings, smartphones and vehicles. The $R^{2}$ of Device A in Vehicle 1 and Device B in Vehicle 2 are almost the same, with the value of as high as about

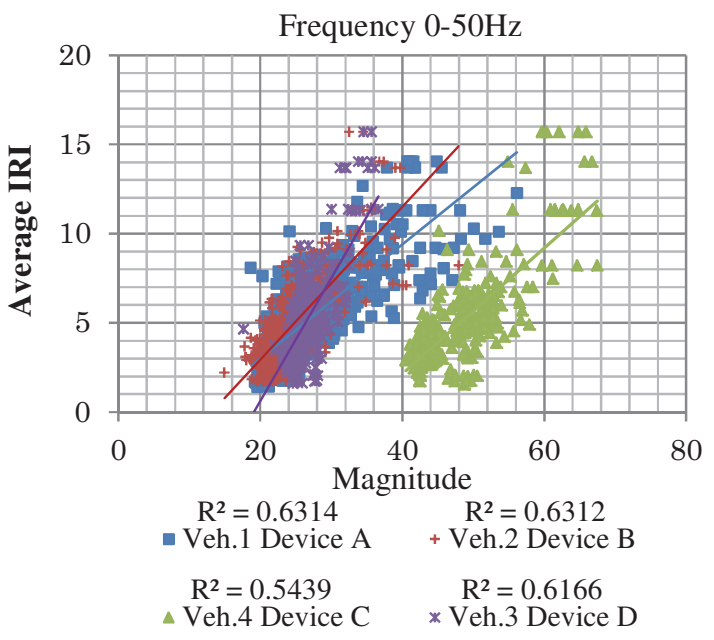

Fig.3 Relationship between road roughness condition (avg. IRI) and acceleration data (magnitude).

Table1 Summary of multiple regression analysis.

\begin{tabular}{|c|c|c|c|c|c|c|c|c|}
\hline & \multicolumn{2}{|c|}{$\begin{array}{l}\text { Device A } \\
\text { Vehicle } 1\end{array}$} & \multicolumn{2}{|c|}{$\begin{array}{l}\text { Device B } \\
\text { Vehicle } 2\end{array}$} & \multicolumn{2}{|c|}{$\begin{array}{l}\text { Device D } \\
\text { Vehicle } 3\end{array}$} & \multicolumn{2}{|c|}{$\begin{array}{l}\text { Device C } \\
\text { Vehicle } 4\end{array}$} \\
\hline Observations & \multicolumn{2}{|c|}{703} & \multicolumn{2}{|c|}{488} & \multicolumn{2}{|c|}{421} & \multicolumn{2}{|c|}{382} \\
\hline Multiple R & \multicolumn{2}{|c|}{0.797} & \multicolumn{2}{|c|}{0.798} & \multicolumn{2}{|c|}{0.758} & \multicolumn{2}{|c|}{0.819} \\
\hline R Square & \multicolumn{2}{|c|}{0.635} & \multicolumn{2}{|c|}{0.638} & \multicolumn{2}{|c|}{0.575} & \multicolumn{2}{|c|}{0.670} \\
\hline Adjusted R Square & \multicolumn{2}{|c|}{0.634} & 0.6 & & \multicolumn{2}{|c|}{0.573} & \multicolumn{2}{|c|}{0.669} \\
\hline \multirow[t]{2}{*}{ F Stat } & \multicolumn{2}{|c|}{609.790} & \multicolumn{2}{|c|}{427.417} & \multicolumn{2}{|c|}{282.786} & \multicolumn{2}{|c|}{385.312} \\
\hline & Coeff & t Stat & Coeff & t Stat & Coeff & $\mathrm{t}$ Stat & Coeff & $t$ Stat \\
\hline Intercept & -2.467 & -5.868 & -4.317 & -7.604 & -21.258 & -12.780 & -23.767 & -14.536 \\
\hline Magnitude & 0.305 & 28.820 & 0.403 & 23.684 & 0.905 & 18.425 & 0.528 & 20.324 \\
\hline Avg. Speed & -0.013 & -2.733 & -0.016 & -2.905 & 0.054 & 5.533 & 0.074 & 7.862 \\
\hline
\end{tabular}

0.6314 and 0.6312 , respectively; while the $R^{2}$ of Device D in Vehicle 3 and Device $C$ in Vehicle 4 are 0.5439 and 0.6166, respectively. Fig. 3 also shows that the magnitude of vibrations for vehicle $\mathrm{C}$, which are placed inside the pocket, is greater than other smartphones that are placed at other locations. To investigate the effect of the speed on the relationship, multiple regression analysis has been carried out. Table 1 summarized multiple regression analysis of the device and vehicle corresponding to the results presented in Fig. 3.

As shown in Table 1, $R^{2}$ improvements have been observed by adding the average speed into the regression. The improvements are slightly in the cases of Device A and Device B, which are the devices that have been fixed, in Vehicle 1 and Vehicle 2, respectively; while the improvements in the cases of the free devices, Device D in Vehicle 3 and Device C in Vehicle 4 , are considerably larger.

Another important finding is that, at the frequency range of $40-50 \mathrm{~Hz}$, the magnitude of acceleration data (derived from frequency domain analysis or FFT) is the most useful in reflecting the vibration caused by road roughness condition. As shown in Fig. 4, Fig. 5 and Fig. 6, the $R^{2}$ for Device $\mathrm{C}$ in Vehicle 1 at the frequency range of $40-50 \mathrm{~Hz}$ is the greatest, significantly greater than at frequency range $20-30 \mathrm{~Hz}$ and $0-10$ $\mathrm{Hz}$, for instances. Additionally, based on the condition indexes, that we have adopted ${ }^{13)}$, similar ten- 


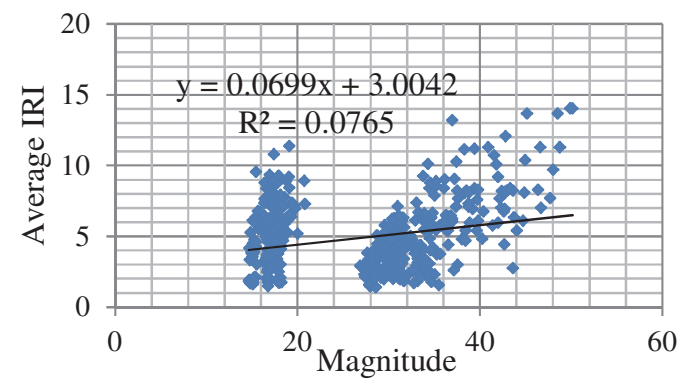

Fig.4 Relationship of Avg. IRI and Magnitude at freq. 0$10 \mathrm{~Hz}$ (Veh.1 Device C).

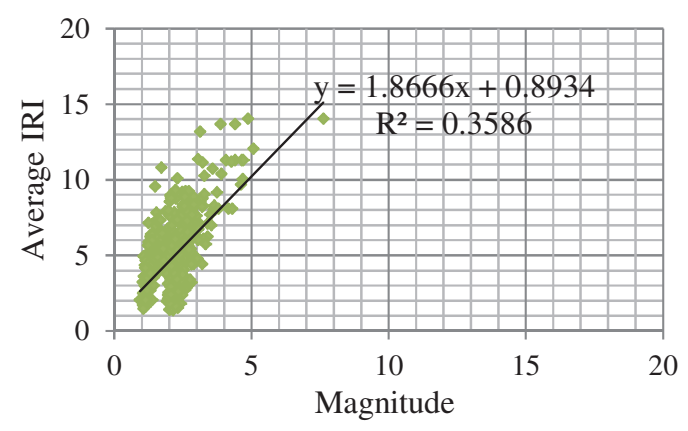

Fig.5 Relationship of Avg. IRI and Magnitude at freq. 20$30 \mathrm{~Hz}$ (Veh.1 Device C).

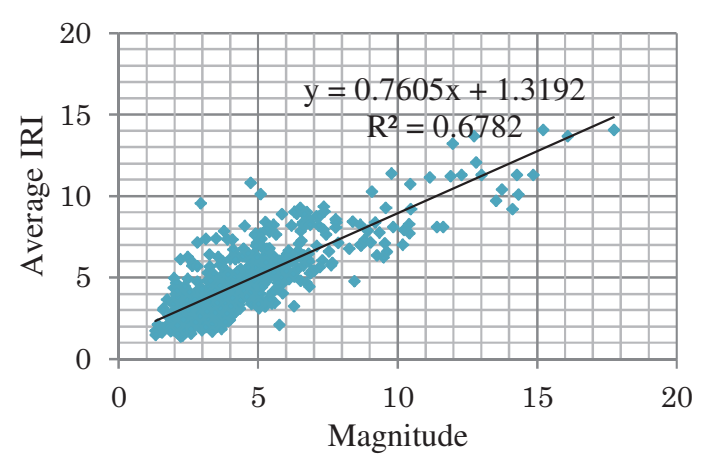

Fig.6 Relationship of Avg. IRI and Magnitude at freq. 40$50 \mathrm{~Hz}$ (Veh.1 Device C).

dency of the classification of the sum of magnitudes of acceleration vibration is also observed.

In conclusion, a simple linear model can be used to estimate IRI from acceleration and speed data. In other words, road roughness condition can be roughly modeled as a function of the acceleration magnitudes and the average speed. However, if the smartphone setting and vehicle type are different; model coefficient parameters may also be different.

The magnitudes at the frequency range of $40-50 \mathrm{~Hz}$ are selected for the formulation of the model.

\section{MODEL FORMULATION}

Assuming we would like to estimate road roughness condition of road sections, within a road network, from a lot of data (acceleration and average speed data) collected by different smartphones, which are placed inside different vehicles (presumably running on the network randomly). From the conclusion that we have found in our previous studies ${ }^{17), 18), 19)}$, as introduced in the chapter above, that the road roughness condition can be roughly modeled as a function of the acceleration magnitudes and the average speed of different smartphones or vehicles. We assume the following function:

$$
C_{k}=A_{0 i}+A_{1 i} M_{i k}+A_{2 i} v_{i k},
$$

where:

$C_{k}:$ Road roughness condition (IRI) at section $k$,

$M_{i k}:$ Magnitude of acceleration in frequency domain for vehicle $i$ at section $k$,

$v_{i k}:$ Average speed of vehicle $i$ at section $k$, $A_{0 i}, A_{1 i}, A_{2 i}$ : Coefficients of road roughness condition function for vehicle $i$.

Note that the parameter $A_{0 i}, A_{1 i}$ and $A_{2 i}$ represent the variation of parameters, which depend on vehicle type, smartphone type and smartphone setting.

The problem here is to estimate the unknown variables $\left(C_{k}, A_{0 i}, A_{1 i}\right.$, and $\left.A_{2 i}\right)$ by minimizing the error of the model. If we consider least squared error regarding $C_{k}$, object function $F$ can be formulated from the relationship in the equation (1):

$$
\begin{aligned}
F=\sum_{i} \sum_{j} \sum_{k} \delta_{i j k} \\
\cdot\left\{C_{k}-\left(A_{0 i}+A_{1 i} M_{i j}^{o b s}+A_{2 i} v_{i j}^{o b s}\right)\right\}^{2}
\end{aligned}
$$

where $j$ is the sequential data of vehicle $i$; and $\delta_{i j k}$ equals to 1 if the data $j$ of vehicle $i$ has passed through section $k$, while $\delta_{i j k}$ equals to 0 if the data $j$ of vehicle $i$ has not passed through section $k . M_{i j}^{o b s}$ and $v_{i j}^{o b s}$ are observed magnitudes and observed average speed, respectively.

Under this formulation, $C_{k}, A_{0 i}, A_{1 i}$, and $A_{2 i}$ can be estimated provided that we have observed IRI data (denoted by $C_{k}^{o b s}$ ) at several observed sections $k \in$ $K_{o b s}$, where $K_{o b s}$ is a set of observed sections, obtaining from a more precise method (such as VIMS in our study). However, minimization of equation (2) under the constraint of $C_{k}=C_{k}^{o b s}$ does not yield appropriate estimators, because, in the minimization, results are largely affected by the unobserved sections, where smaller values of $C_{k}, A_{0 i}, A_{1 i}$, and $A_{2 i}$ result in smaller value of equation (2).

Therefore, we propose a method as the following steps:

1) First, we introduce a relative relationship of the road roughness condition.

$$
c_{k}=a_{0 i}+a_{1 i} M_{i k}+a_{2 i} v_{i k}
$$

where $c_{k}$ is the relative road roughness condition at section $k ; a_{0 i}, a_{1 i}$ and $a_{2 i}$ are coefficients of relative 
road roughness condition function for vehicle $i$. Note that equation (3) can be derived by dividing both sides of equation (1) by the same value.

2) Formulate objective function $f$, which is least square error regarding $c_{k}$ under a constraint level of value for parameter $a_{1 i}$ :

$$
\begin{gathered}
f=\sum_{i} \sum_{j} \sum_{k} \delta_{i j k}\left\{c_{k}-\left(a_{0 i}+a_{1 i} M_{i j}^{o b s}+a_{2 i} v_{i j}^{o b s}\right)\right\}^{2} \\
\text { s.t. } \quad \frac{1}{N} \sum_{i} a_{1 i}=1
\end{gathered}
$$

where $N$ is the number of vehicles.

3) Estimate $c_{k}^{e s t}, a_{0 i}^{e s t}, a_{1 i}^{e s t}$, and $a_{2 i}^{e s t}$, these are $c_{k}, a_{0 i}, a_{1 i}$ and $a_{2 i}$, respectively so as to minimize objective function $f$ in equation (4).

4) Assume $C_{k}=b_{0}+b_{1} c_{k}$, where $b_{0}$ and $b_{1}$ are parameters that would be derived from solving the regression of $C_{k}^{o b s}=b_{0}+b_{1} c_{k}^{e s t}\left(k \in K_{o b s}\right)$. In order words, $b_{0}$ and $b_{1}$ are calculated from observed road sections that have precise IRI values.

Then, road roughness condition $C_{k}^{e s t}$ can be estimated applying the function $C_{k}^{e s t}=b_{0}+b_{1} c_{k}^{e s t}$ for all the remaining sections.

After that $A_{0 i}, A_{1 i}$, and $A_{2 i}$ can also be estimated by minimizing the following equation (5) in $A_{0 i}, A_{1 i}$, and $A_{2 i}$ :

$$
\begin{aligned}
F=\sum_{i} \sum_{j} \sum_{k} \delta_{i j k} \\
\cdot\left\{C_{k}^{e s t}-\left(A_{0 i}+A_{1 i} M_{i j}^{o b s}+A_{2 i} v_{i j}^{o b s}\right)\right\}^{2}
\end{aligned}
$$

\section{NUMERICAL EXAMPLES}

The aim of the numerical examples is to validate the model performance in some simple cases.

\section{(1) Setting}

In our setting, we assume the road network that consists of 18 links (same length) and each link is set to have 20 sections (same length), see Fig. 7. Road roughness condition is set to be good for all sections in link 8 and 11; fair for all section in link 3, 4, 5, 6, 13, 14, 15 and 16; poor for all sections in link 1, 2, 7, 10, 17 and 18; and finally, bad for all sections in link 9 and 12. In other words, IRI for each section is assigned randomly within an average IRI range corresponding to its condition index (good, fair, poor, and bad). See Table 2.

Note that the condition index presented in Table 2 is adopted from the condition index of the Road Management System used in $\operatorname{Laos}^{20)}$.

Other settings include: route arrangement (see Table 3 and Fig. 8) and vehicle settings (detailed in Fig. 9).

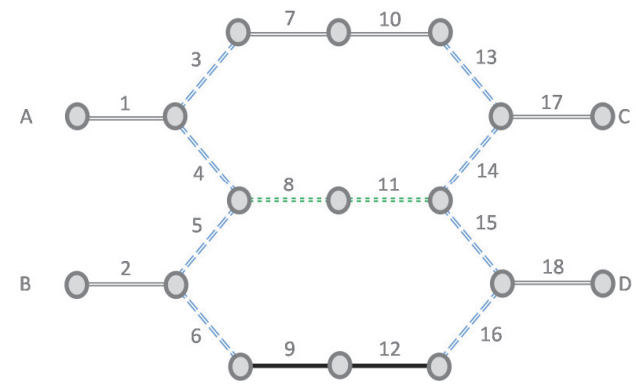

Fig.7 Road network.

Table2 Road condition index.

\begin{tabular}{c:c:c:c}
\hline Condition Index & Average IRI & Road Link & Legend \\
\hline Good & $0 \leq \mathrm{IRI}<4$ & 8,11 & 0 \\
Fair & $4 \leq \mathrm{IRI}<7$ & $3,4,5,6,13,14,15,16$ & 0 \\
Poor & $7 \leq \mathrm{IRI}<10$ & $1,2,7,10,17,18$ & 0 \\
Bad & $\mathrm{IRI} \geq 10$ & 9,12 & 0 \\
\hline
\end{tabular}

Table3 Route arrangement.

\begin{tabular}{c:c|}
\hline Route & Via Links \\
\hline A & $1,4,8,11,14$, and 17 \\
B & $1,3,7,10,13$, and 17 \\
C & $2,5,8,11,15$, and 18 \\
D & $2,6,9,12,16$, and 18 \\
E & $1,4,8,11,15$, and 18 \\
F & $2,5,8,11,14$, and 17 \\
\hline
\end{tabular}

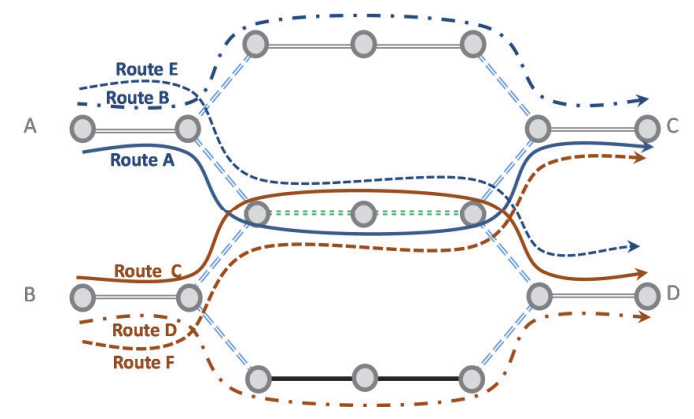

Fig.8 Map of route arrangement.

\section{(2) Methodology}

The simulation processes are summarized in Fig. 10.

Details of the 5 scenarios are:

i. Only link 8 and 11 (all good condition) are observed precisely, the remaining links are observed roughly with errors;

ii. Only link 9 and 12 (all bad condition) are observed precisely, the remaining links are observed roughly with errors;

iii. Only link 8 and 9 (one good condition and one bad condition) are observed precisely, the remaining links are observed roughly with errors;

iv. Only link 7, 8, 9 and 14 (good, fair, poor and bad; one link each) are observed precisely, the remaining links are observed roughly with errors;

v. Only link 1, 5, 11 and 12 (good, fair, poor and 
bad; one link each) are observed precisely, the remaining links are observed roughly with errors.

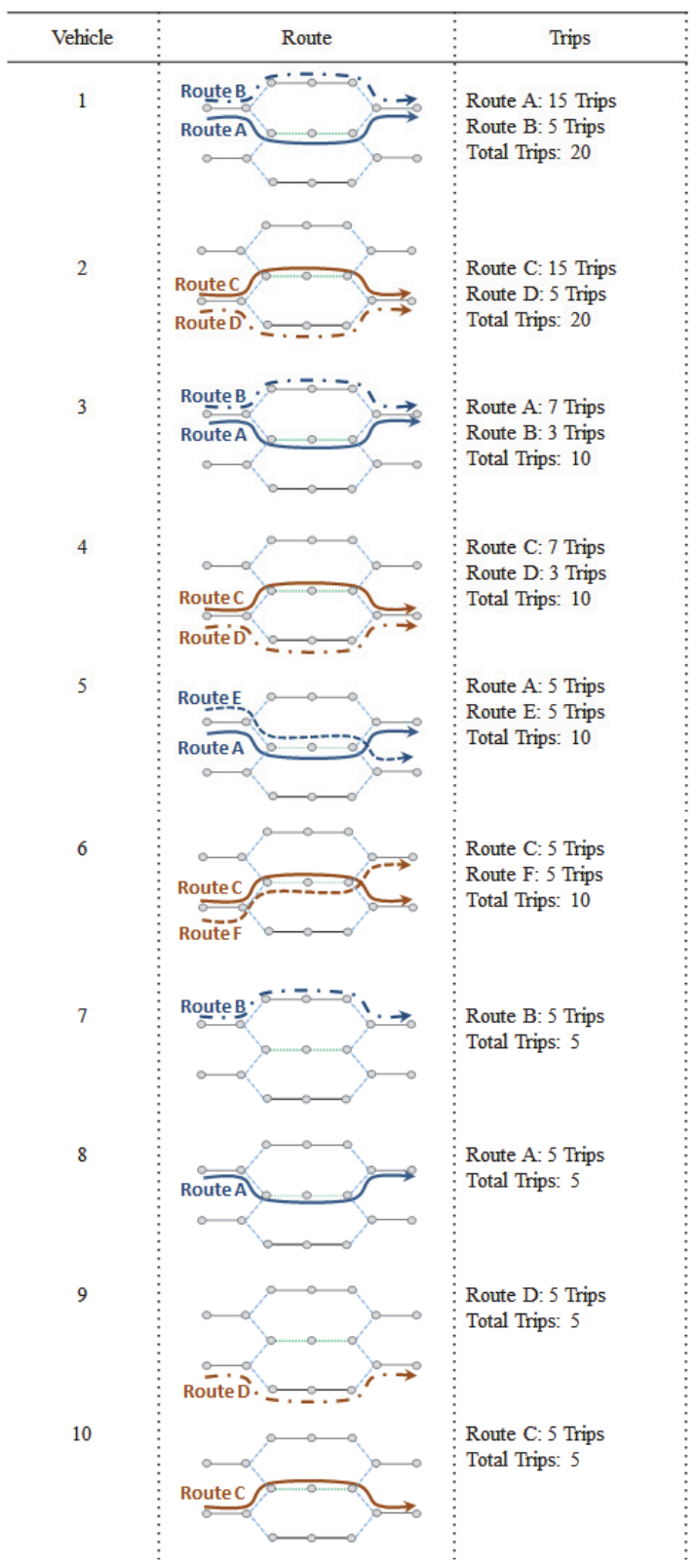

Fig.9 Vehicle settings.

Assume real IRI $\left(C_{k}{ }^{\text {real }}\right)$ for each section

Assume vehicle $i$ with average speed for each $k\left(v_{i k}\right)$ Assume magnitudes for each $i$ and $k\left(M_{i k}\right)$

- 5 scenarios are considered

Assume observed IRI ( $\left.C_{k}^{o b s}\right)$ for each section under the different scenarios

Estimate the relative road roughness condition $\left(c_{k}{ }^{\text {est }}\right)$ Calculate the estimated IRI ( $\left.C_{k}^{\text {est }}\right)$ from the relative road roughness condition $\left(c_{k}{ }^{e s t}\right)$

- Compare the estimated IRI $\left(C_{k}{ }^{e s t}\right)$ and the real IRI $\left(C_{k}^{\text {real }}\right)$

Fig.10 Simulation processes.

\section{(3) Results}

After running the simulations for 5 cases as mentioned above, the performance of the model can be summarized in the following figures:

a) Scenario 1: only link 8 and 11 (all good condition links) are observed precisely (Fig. 11).

b) Scenario 2: only link 9 and 12 (all bad condition links) are observed precisely (Fig. 12).

c) Scenario 3: only link 8 and 9 (one good condition link and one bad condition link) are observed precisely (Fig. 13).

d) Scenario 4: only link 7, 8, 9 and 14 (good, fair, poor and bad condition links, respectively) are observed precisely (Fig. 14).

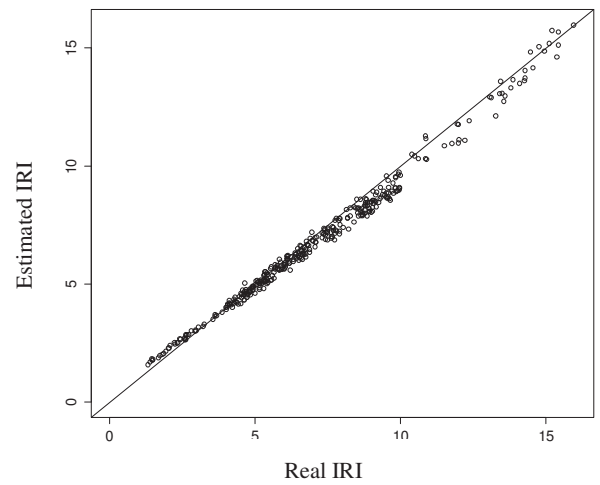

Fig.11 Real and estimated IRI (Scenario 1).

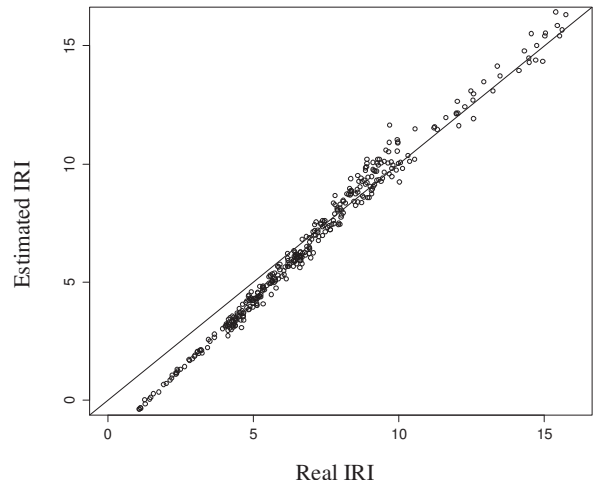

Fig.12 Real and estimated IRI (Scenario 2).

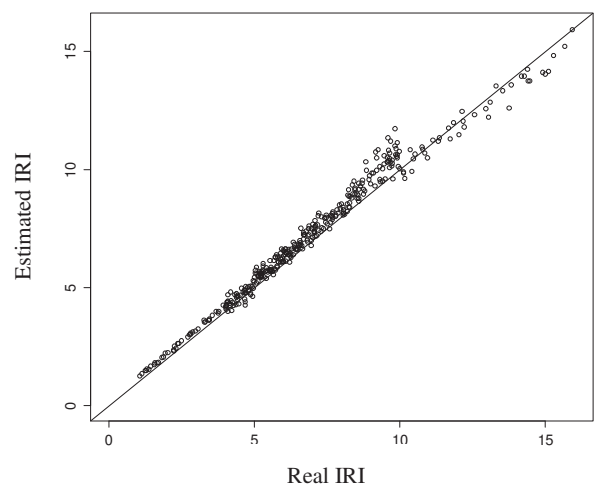

Fig.13 Real and estimated IRI (Scenario 3). 


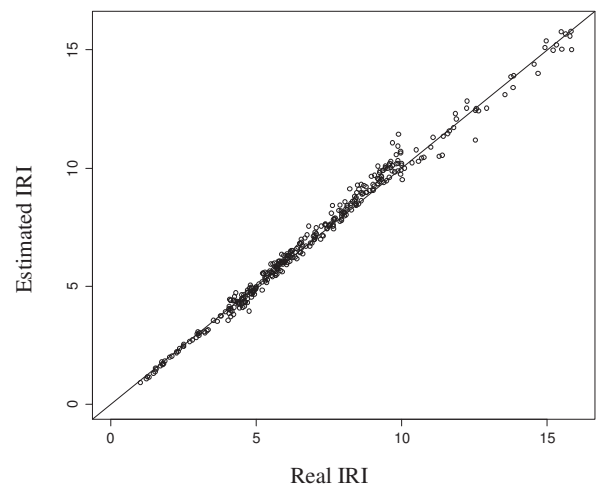

Fig.14 Real and estimated IRI (Scenario 4).

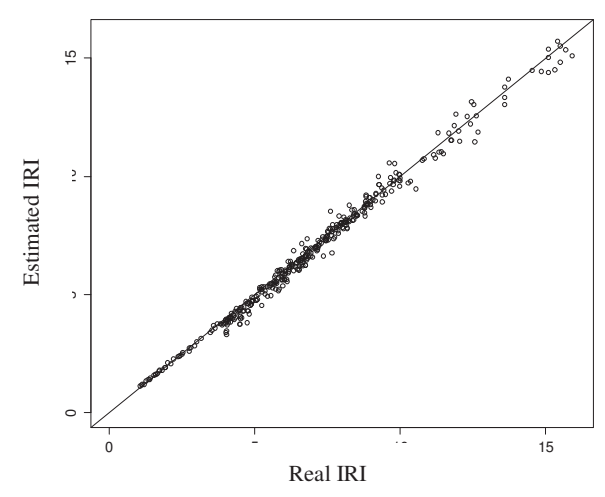

Fig.15 Real and estimated IRI (Scenario 5).

e) Only link 1, 5, 11 and 12 (good, fair, poor and bad condition links, respectively) are observed precisely (Fig. 15).

\section{(4) Discussion}

In general, the model performs considerably good as it yields very good fitting for all the simulation cases (Fig. 11 to Fig. 15 above). In fact, the model obviously perform better, as shown in Fig. 14 and Fig. 15, if different type of road roughness conditions, a mix of all condition ranging from good, fair, poor and bad, are considered as the observed road roughness condition (IRI). Statistic indicator (the Root Mean Squared Error, RMSE) also shows that the performances of the model in the cases of scenario 4 and 5 , which consider all different types of road condition as observed links, are much better than the cases of the remaining scenarios. The average Percentage Root Mean Squared Error (PRMSE) of scenario 4 and 5 (4.7\% and $4.6 \%$ respectively) are considerably very low when compare to $10 \%$ of the error assumed in our simulation. The prediction in the cases of scenario 4 and scenario 5 also appears to be more reliable than other scenarios judging from their RMSE or PRMSE range widths, which are very small. See Table 4.
Table4 Model performance of each scenario derived from a total of 5 runs.

\begin{tabular}{|c|cc|c|cc|c|}
\hline \multirow{2}{*}{ Scenario } & \multicolumn{3}{|c|}{ RMSE } & \multicolumn{3}{c|}{ PRMSE } \\
\cline { 2 - 7 } & Range & Average & Range Width & Range & Average & Range Width \\
\hline \hline 1 & $0.368-0.757$ & 0.5 & 0.389 & $0.052-0.107$ & 0.071 & 0.055 \\
2 & $0.494-1.328$ & 0.889 & 0.834 & $0.071-0.191$ & 0.128 & 0.12 \\
3 & $0.214-0.495$ & 0.36 & 0.281 & $0.031-0.071$ & 0.052 & 0.04 \\
4 & $0.258-0.415$ & 0.324 & 0.157 & $0.037-0.06$ & 0.047 & 0.023 \\
\hline 5 & $0.283-0.375$ & 0.325 & 0.092 & $0.04-0.054$ & 0.046 & 0.014 \\
\hline
\end{tabular}

Table5 Vehicle/Device setting combinations.

\begin{tabular}{|c|c|c|c|}
\hline $\begin{array}{c}\text { Combination } \\
\text { Number }\end{array}$ & $\begin{array}{c}\text { Combination } \\
\text { Code }\end{array}$ & Description & Remarks \\
\hline 1 & $A V 1$ & Device A (dash board) Vehicle 1 & Selected \\
\hline 2 & AV2 & Device A (dash board) Vehicle 2 & Selected \\
\hline 3 & AV3 & Device A (dash board) Vehicle 3 & Selected \\
\hline 4 & AV4 & Device A (dash board) Vehicle 4 & Selected \\
\hline 5 & BV1 & Device B (dash board) Vehicle 1 & Excluded \\
\hline 6 & BV2 & Device B (dash board) Vehicle 2 & Excluded \\
\hline 7 & BV3 & Device B (dash board) Vehicle 3 & Excluded \\
\hline 8 & BV4 & Device B (dash board) Vehicle 4 & Excluded \\
\hline 9 & CV1a & Device C (inside pocket) Vehicle 1 & Selected \\
\hline 10 & CV1b & Device C (box near gear handle) Vehicle 1 & Selected \\
\hline 11 & CV2 & Device C (inside pocket) Vehicle 2 & Selected \\
\hline 12 & CV3 & Device C (inside pocket) Vehicle 3 & Selected \\
\hline 13 & CV4 & Device C (inside pocket) Vehicle 4 & Selected \\
\hline 14 & DV1a & Device D (inside pocket) Vehicle 1 & Selected \\
\hline 15 & DV $1 b$ & Device D (box near gear handle) Vehicle 1 & Selected \\
\hline 16 & DV2 & Device D (inside pocket) Vehicle 2 & Selected \\
\hline 17 & DV3 & Device D (inside pocket) Vehicle 3 & Selected \\
\hline 18 & DV4 & Device D (inside pocket) Vehicle 4 & Selected \\
\hline
\end{tabular}

\section{THE ESTIMATION USING REAL SEN- SOR DATA}

To further validate that the model, formulated and tested in the numerical simulations above, is actually sufficient to be used for road surface roughness estimation from smartphone sensor data, an example using real sensor data from our experiment are carried out.

From the experiment, 18 unique combinations of vehicle/device setting are available (the combinations of 4 vehicles and 4 devices setting), however due to some technical issues concerning the GPS data and time constraint, data of one device (Device B) have been excluded, therefore only 14 unique combinations are selected for the analysis in this example (Table 5).

A total of 1,865 of road sections have been selected from the experiment (Fig. 16). At each of the total 1,865 road sections, at least 1 or more unique combinations of vehicle/device setting have passed through once or multiple times. At some sections, there are more than one combination and repetitions. For each combination, we have average speed, magnitudes from FFT and average IRI at each section. Totally there are 5,685 observations for this example.

In the numerical simulations above, we have discussed that selecting a mix of different road section with different conditions would allow us to predict road roughness condition more accurately and reliable. Therefore, in this example we select 37 different road sections, which equal to approximately $2 \%$ of the total road sections, with different road conditions as observed sections with known IRI values. These 


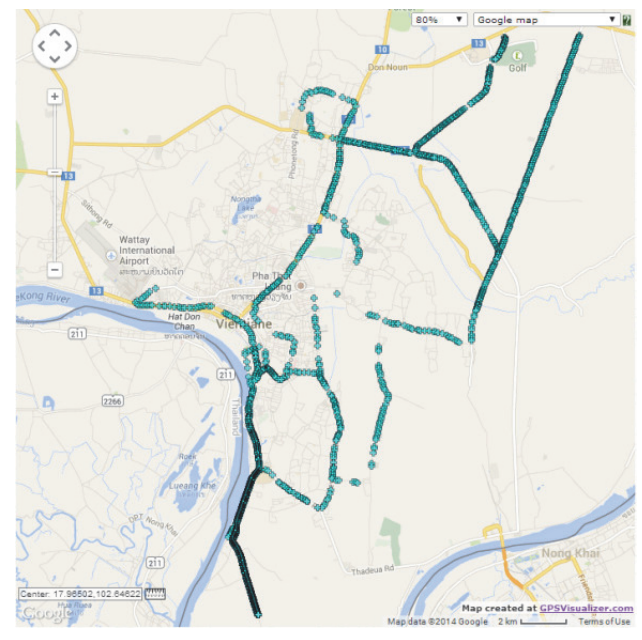

Fig.16 Selected road sections.

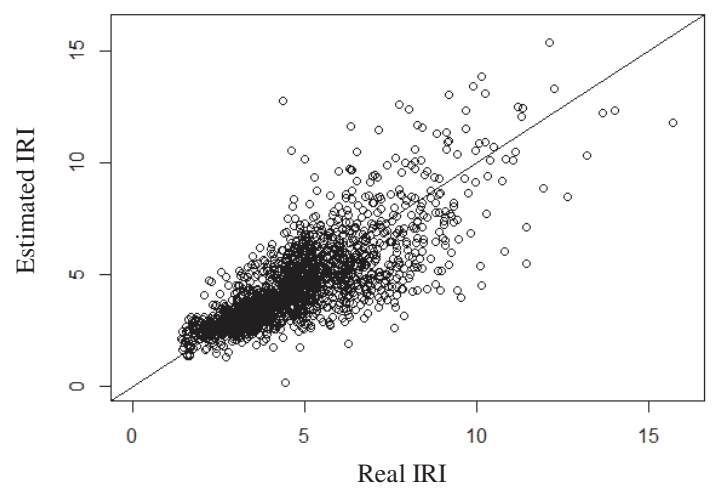

Fig.17 Real and estimate IRI, Real Data Example.

observed sections are used to estimate the parameter coefficients where they will be then used in the model, also discussed above, to estimate IRI for other sections.

After running the simulation, the result of this example shows that by using $2 \%$ of the total number of road sections, the estimate model can predict IRI of the remaining road sections with approximately $29 \%$ of errors (PRMSE of 0.290). See Fig. 17.

\section{CONCLUSION}

In this study, we develop a model to estimate road roughness condition (IRI). In order to realize the model, it is necessary to understand the relationship between road roughness condition and the data to be obtained using smartphone devices. We have carried out an experiment to collect the data (acceleration and speed) using Android smartphones. The analysis, which includes matching of data with reference data and investigation in frequency domain, proofs that road roughness condition is a linear function of magnitude of acceleration and average speed.

After model formulation, numerical examples have been simulated. The simulation results show that the model performs promisingly, particularly in cases, where observed road roughness condition consists of different IRI values spreading across different road condition indexes. Additionally an example using real sensor data is also performed. The result of the example confirms that the simple model we developed can be used to predict IRI of road surface from smartphone sensor data.

In our future works, more focus will be put into improving the model, data collection piloting and practical application.

\section{REFERENCES}

1) Sayer, M. W., Gillespie, T. D. and Queiros, C. A. V. : International Road Roughness Experiment, The World Bank, 1986. Available online at: http:// deepblue.lib.umich.edu/bitstream/handle/ 2027.42/3134/72773.pdf; jsessionid= 2D55BF78AABCA31452E6A59CD28D17C1? sequence $=2$ [Accessed Sept. 2012]

2) González, A., O’brien, E. J., Li, Y. Y. and Cashell, K. : The use of vehicle acceleration measurements to estimate road roughness, Vehicle System Dynamics, Vol. 46, No. 6, pp. 483-499, 2008.

3) Eriksson, J., Girod, L., Hull, B., Newton, R., Madden, S. and Balakrishnan, H. : The pothole patrol: using a mobile sensor network for road surface monitoring, Paper presented at the Sixth International Conference on Mobile System, Applications and Services, Breckenridge, Colorado, United States, June 17-20, 2008.

4) Mohan, P., Padmanabhan, V. N. and Ramjee, R. : Nericell: Rich Monitoring of Road and Traffic Condition using Mobile Smartphones, Proc. of the $6^{\text {th }}$ ACM Conference on Embedded Network Sensor Systems, pp. 323-336, 2008.

5) Mednis, A., Strazdins, G., Zviedris, R., Kanonirs, G. and Selavo, L. : Real time pothole detection using Android smartphones with accelerometers, Paper presented at the 2011 International Conference on Distributed Computing in Sensor Systems, Barcelona, Spain, June 27-29, 2011.

6) Strazdins, G., Mednis, A., Kanonirs, G., Zviedris, R. and Selavo, L. : Towards Vehicular Sensor Networks with Android Smartphones for Road Surface Monitoring, Paper presented at the $2^{\text {nd }}$ International Workshop on Networks of Cooperating Objects, Chicago, USA, April 11, 2011.

7) Tai, Y., Chan, C. and Hsu, J. Y. : Automatic road anomaly detection using smart mobile device, Paper presented at the 2010 Conference on Technologies and Applications of Artificial Intelligence, Hsinchu, Taiwan, November 18-20, 2010.

8) Perttunen, M., Mazhelis, O., Cong, F., Kauppila, M., Leppänen, T., Kantola, J., Collin J., Pirttikangas, S., Haverinen, J. and Ristaniemi, T. : Distributed road surface condition monitoring using mobile phones, Ubiquitous Intelligence and Computing, pp. 64-78, 2011.

9) Android Application: BumpRecorder, Google Play Store, Available online at: https://play.google.com/store/ apps/details?id=jp.traffichazard. 
BumpRecorder\&hl=en [Accessed Feb. 2013]

10) Android Application: Roadroid, Available online at: http://www.roadroid.se/ [Accessed Jan. 17, 2014].

11) Fujino, Y., Kitagawa, K. and Furukawa, T. : Development of vehicle intelligent monitoring system (VIMS), Proc. of SPIE, Vol. 5765, pp. 148-157, 2005.

12) Furukawa, T., Fujino, Y., Kubota, K. and Ishii, H. : Real-time diagnostic system for pavements using dynamic response of road patrol vehicles (VIMS), Proc. 3rd International Conference on Structural Health Monitoring of Intelligent Infrastructure (SHMII-3), 2007.

13) Vehicle Intelligent Monitoring System : VIMS, Bridge and Structure Laboratory, the University of Tokyo, Japan, http://www.bridge.t.u-tokyo . ac. jp/NHM]2005/7.pdf [Accessed Apr. 2, 2012]

14) Nagayama, T., Miyajima, A., Kimura, S., Shimada, Y. and Fujino, Y. : Road condition evaluation using the vibration response of ordinary vehicles and synchronously recorded movies, Proc. of SPIE, Vol. 8692, pp. 86923A1-12, 2013.

15) Android Application: AndroSensor, Google Play Store, Available online at: https://play.google.com/store/apps/ details?id=com. fivasim. androsensor\&hl=en [Accessed Sept. 2012]

16) VIMS Manual, VIMS Consortium, Nagasaki University, Japan, 2012.

17) Douangphachanh, V. and Oneyama, H. : A Study on the Use of Smartphones for Road Roughness Condition Estimation, Proc. of the Eastern Asia Society for Transportation Studies, P297, Vol. 9, 2013; and J. Eastern Asia Society for Transport Studies, Vol. 10, pp. 1551-1564, 2013.

18) Douangphachanh, V. and Oneyama, H. : Using Smartphones to Estimate Road Pavement Condition, Paper presented at the 1st International Symposium for Next Generation Infrastructure, Wollongong, NSW, Australia, October 1-4, 2013.

19) Douangphachanh, V. and Oneyama, H.: Estimation of Road Roughness Condition from Smartphones under Realistic Settings, Proc. of the $13^{\text {th }}$ International Conference on ITS Telecommunication (ITST), pp. 427433, 2013.

20) PTI : Lao Road Management System, 2011.

(Received February 28, 2014) 